\title{
Enhancement of the Adhesive Properties by Optimizing the Water Content in PNIPAM-Functionalized Complex Coacervates
}

Marco Dompé, Mehdi Vahdati, Froukje van Ligten, Francisco J. Cedano-Serrano, Dominique Hourdet, Costantino Creton, Marco Zanetti, Pierangiola Bracco, Jasper van der Gucht, Thomas Kodger, and Marleen Kamperman*

Cite This: ACS Appl. Polym. Mater. 2020, 2, 1722-1730

Read Online

ACCESS | Llll Metrics \& More | 回 Article Recommendations | sl Supporting Information

ABSTRACT: Most commercially available soft tissue glues offer poor performance in the human body. We have developed an injectable adhesive whose setting mechanism is activated by a change in environmental factors, i.e., temperature and/or ionic strength. The material and setting process are inspired by the adhesive processing mechanism observed in natural maritime glues. Complex coacervation, a liquid-liquid phase separation

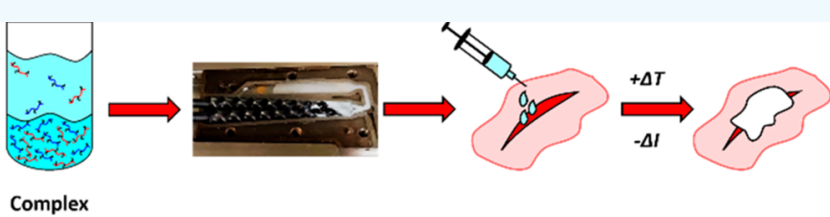
Coacervate Adhesive

Extrusion

Liquid Adhesive

Tough Solid between oppositely charged polyelectrolytes, is thought to play an important role in the processing. Complex coacervates are characterized by a high water content, which inevitably weakens the glue. Here, we aim to increase the adhesive performance by systematically tuning the water content. Among the several strategies here explored, the most effective one is the mechanical removal of water using an extruder, resulting in an increase of work of adhesion by 1 order of magnitude compared to the original formulation.

KEYWORDS: complex coacervation, water content, underwater adhesion, extrusion, bioinspired adhesives

\section{INTRODUCTION}

Adhesive technology, despite being known and exploited since the middle Pleistocene, ${ }^{1}$ is rarely applied when dealing with adverse environments. In medicine, for instance, surgical tissue closure still relies on conventional techniques, such as suturing and stapling, ${ }^{2}$ which have many pitfalls, e.g., inflammatory reactions, scar formation, and stress localization. ${ }^{3-5}$ Tissue glues represent a valid alternative to these methods, especially because of the easy handling and minimal tissue damage. $3,5,6$ However, most commercially available adhesives fail to offer a proper performance in wet and dynamic environments and do not achieve the required bonding strength.

In nature, many aquatic species (e.g., sandcastle worms and mussels) have managed to solve the challenges related to underwater adhesion by developing protein-based glues that bond strongly to a variety of surfaces even in submerged conditions. $^{8-10}$ A phenomenon directly involved in the processing and delivery of these protein-based adhesives is complex coacervation, ${ }^{1,12}$ an associative phase separation process which requires the presence of two oppositely charged polyelectrolytes. ${ }^{13}$ Under the proper conditions $(\mathrm{pH}$, ionic strength, and mixing ratio), the polyelectrolyte solution partitions into a dilute phase (polymer-poor) and a complex coacervate phase (polymer-rich). ${ }^{14}$ Several research groups have employed these electrostatic interactions to fabricate viscous glues, most of which set underwater due to covalent cross-linking reactions, providing the strength required to oppose detachment. ${ }^{15-20}$
Previously, ${ }^{21-23}$ we have produced a complex coacervatebased adhesive which undergoes a liquid-to-solid transition in response to environmental triggers, forming exclusively physical bonds without the addition of any cross-linking agent. The glue is prepared by mixing oppositely charged polyelectrolytes grafted with thermoresponsive poly $(\mathrm{N}$ isopropylacrylamide) (PNIPAM) chains. PNIPAM is a water-soluble polymer which phase separates at temperatures above its lower critical solution temperature (LCST), which is around $32{ }^{\circ} \mathrm{C}$. The adhesive can be reinforced by heating the sample above the LCST (temperature switch), by immersing the sample in a lower ionic strength medium (salt switch), or by combining the two triggers (temperature + salt switch), mimicking the conditions that the glue would experience in the human body. However, despite optimizing the performance by assessing the effect of different parameters (polymer composition, temperature, ionic strength and probe surface), we could obtain, at best, a 3-fold enhancement of the adhesive properties compared to the original formulation. ${ }^{21-23}$

Received: March 2, 2020

Accepted: March 20, 2020

Published: March 20, 2020 
For this adhesive system, and basically for all watercontaining adhesives, the adhesive performance and the mechanical properties heavily depend on water content. For instance, commercial poly(ethylene glycol) (PEG)-based glues, such as DuraSeal and CoSeal, bind to tissues with a low adhesive strength, which is mainly attributable to the high water content, ranging from $90 \%$ to $99 \% \mathrm{w} / \mathrm{v}^{24}$ Another related drawback is the significant swelling in physiological conditions $(>700 \%),{ }^{25}$ which can lead to medical complications and further weakening of the adhesive: DuraSeal has been reported to cause nerve compression, ${ }^{26,27}$ while CoSeal has shown a dramatic decrease in both moduli and energy to failure (in compression mode) over a period of 3 days in physiological conditions due to water sorption. ${ }^{28}$ To circumvent issues associated with hydrophilic polymers, relatively hydrophobic surgical glues have been proposed. For example, Karp and co-workers designed a hydrophobic prepolymer (poly(glycerol sebacate acrylate) (PGSA)) which can be crosslinked in situ by UV light: the glue showed limited swelling in physiological conditions, providing stronger adhesion than standard tissue adhesives in highly dynamic environments. ${ }^{29,30}$

However, it is not always desirable to increase the hydrophobicity. For instance, recently Yuk et al. developed dry double-sided tapes whose adhesion mechanism is initiated by the removal of the interfacial water present at the tissue surface: in this case, water absorption by the adhesive matrix is considered a crucial requirement to promote an intimate contact with the tissue, resulting in the quick establishment of both covalent and noncovalent interactions with the surface. ${ }^{31}$ Additionally, water may also act as plasticizer, which can improve the adhesion performance. ${ }^{32}$ Feldstein et al. studied how water content affects the adhesive properties of poly $(\mathrm{N}$ vinylpyrrolidone)-poly(ethylene glycol) (PVP-PEG) blends. ${ }^{33,34}$ By increasing the content of PEG, the water content increased and adhesion was enhanced, enabling the material to sustain higher deformations without considerably affecting the ultimate tensile strength. The optimal performance, in terms of peel force, was observed when the PEG concentration was increased to $36 \%$, with the mode of failure transitioning from adhesive to cohesive, allowing fibrillation within the material. A further increase in water sorption, however, caused a dilution of the entanglement structure, excessively lowering the modulus and making the material too fluid-like, leading to a decrease in adhesion. ${ }^{32}$ Analogous to the work of Feldstein, ${ }^{32-34}$ we believe that the optimization of the water content and, consequently, of the polymer concentration is necessary to enhance the adhesion performance.

In the PNIPAM-based adhesive system that we recently developed, ${ }^{21-23}$ the water content is always above $90 \%$, strongly limiting the adhesive properties. In this work, we test several strategies to reduce and control the water content within the complex coacervate phase, with the goal of enhancing the work of adhesion. The first strategy is by changing the ionic strength: a lower salt concentration results in a higher polymer concentration in the complex coacervate phase. ${ }^{14}$ The second strategy entails increasing the polymer concentration of the solutions when preparing the complex coacervates. The third strategy involves the use of mechanical force to squeeze the water out of the complex coacervate phase by using an extruder. Earlier research has shown that the high shear forces experienced by polyelectrolyte complexes during extrusion are effective in removing liquids trapped within pores, resulting in an enhancement of the mechanical properties. $^{35-37}$

\section{RESULTS AND DISCUSSION}

The polymers used in this work are poly(acrylic acid)-graftedpoly $(N$-isopropylacrylamide) (PAA-g-PNIPAM) and poly(dimethylaminopropylacrylamide)-grafted-poly( $\mathrm{N}$-isopropylacrylamide) (PDMAPAA-g-PNIPAM). The synthesis and characterization procedure is described in an earlier publication, $^{21}$ and in the Supporting Information the composition of the polymers used (Figures S1-S3) and of the samples (Table S2) is given.

Strategy 1: Effect of Salt Concentration. The first explored strategy to reduce the water content is to decrease the salt concentration at preparation conditions. Previous work has indeed shown that complex coacervates retain less water when prepared at low ionic strength due to the stronger electrostatic interactions. ${ }^{14}$ Figure $1 \mathrm{~A}$ shows the water content of the
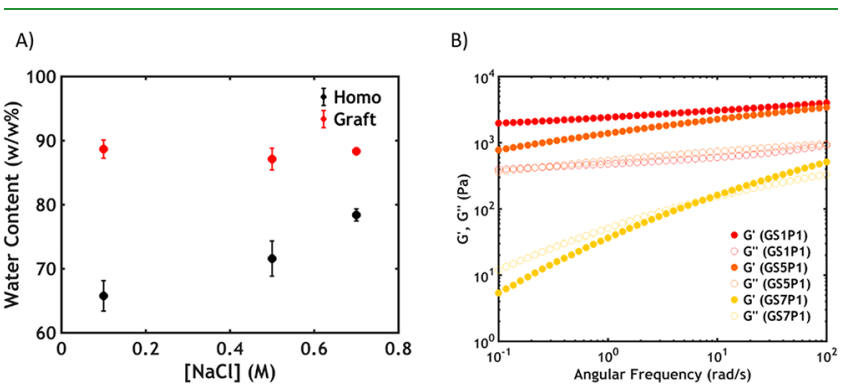

Figure 1. (A) Water content of complex coacervates plotted as a function of the added salt concentration for the samples analyzed in Strategy 1. (B) Frequency sweeps $\left(G^{\prime}\right.$ represented as full dots and $G^{\prime \prime}$ as hollow dots) for graft copolymer complex coacervates below the $\operatorname{LCST}\left(T=5{ }^{\circ} \mathrm{C}\right)$.

analyzed samples as a function of the added salt concentration at $T=20{ }^{\circ} \mathrm{C}$ (no change in water content is observed as a function of temperature). ${ }^{21,23}$

In line with previous reports, ${ }^{14}$ complex coacervates prepared from homopolymer solutions have a lower water content at lower ionic strength. However, the complex coacervates prepared from graft copolymers show a constant water content over the whole range of salt concentrations analyzed. As observed in our previous work, ${ }^{23}$ the constant water content might be ascribed to a different distribution of water among the domains: the excess water could be absorbed, at low ionic strength, by the PNIPAM domains and, at high ionic strength (when PNIPAM chains are more prone to dehydration), ${ }^{38}$ by the polyelectrolyte matrix. Additionally, while homopolymer complex coacervates are transparent or slightly turbid at every salt concentration, graft copolymer complex coacervates turn white at $0.5 \mathrm{M} \mathrm{NaCl}$ and below. At low ionic strength, the formation of a microporous structure, due to the water entrapment within the material, is observed: the higher opacity is attributed to the greater dimensions of the pores, which have sizes big enough to scatter light. ${ }^{39}$

However, even though the water content does not change significantly upon lowering the salt concentration, the mechanical properties are strongly affected, as shown in Figure 1B. At high ionic strength (GS7P1) the material exhibits characteristics of a viscoelastic fluid (Figure 1B), with both storage $\left(G^{\prime}\right)$ and loss $\left(G^{\prime \prime}\right)$ moduli frequency dependent. A crossover between $G^{\prime}$ and $G^{\prime \prime}$ is observed at a characteristic 
frequency $\omega_{\mathcal{c}}$ from which it is possible to calculate the relaxation time $\tau\left(1 / \omega_{\mathrm{c}}\right)$ : at time scales longer than $\tau\left(\omega<\omega_{\mathrm{c}}\right)$ the polymer chains can relax the applied stress by sliding along each other, while at shorter time scales $\left(\omega>\omega_{c}\right)$ the chain movement is partially inhibited, leading to a solid-like response. When lowering the salt concentration, a liquid-tosolid transition is already observed at temperatures below the LCST of PNIPAM: both moduli display a very weak frequency dependence, with $G^{\prime}$ always higher than $G^{\prime \prime}$, which indicates gel formation. This behavior is due to the stronger electrostatic interactions at lower ionic strength, which slow down the relaxation of the polyelectrolyte chains. ${ }^{40}$ This also leads to a high complex viscosity at low ionic strength (Figure S4), which makes it difficult for the sample to be injected through a smallbore needle during application. Therefore, since the water content is independent of the salt concentration, lowering the ionic strength is not an effective strategy to increase the polymer content within the adhesive matrix.

Strategy 2: Effect of Starting Polymer Concentration. The second strategy consists in increasing the polymer concentration upon mixing. Figure 2 shows a schematic phase diagram of the graft copolymer mixture, plotting the added salt concentration as a function of the polymer concentration.
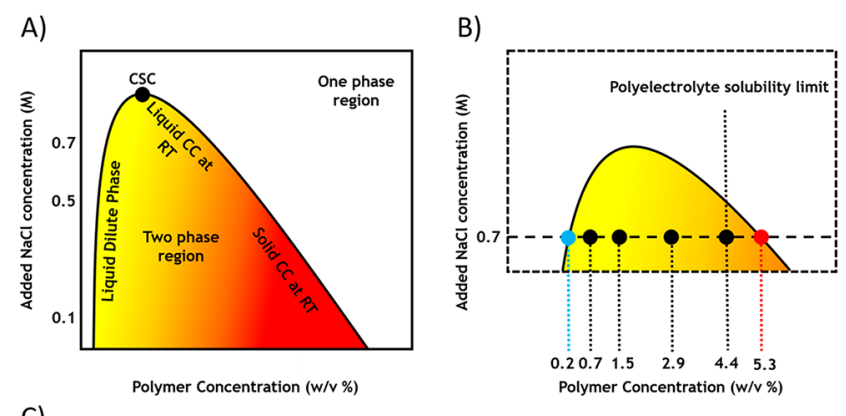

C)

\begin{tabular}{|c|c|c|c|c|}
\hline Sample Name & Used Polymers & $\begin{array}{c}\text { [Added NaCl] } \\
\text { (M) }\end{array}$ & $\begin{array}{c}\text { [Total Charged } \\
\text { Monomer] (M) }\end{array}$ & $\begin{array}{c}\text { Total Polymer } \\
\text { Concentration } \\
\text { (w/v \%) }\end{array}$ \\
\hline GS7P1 & G- \& G+ & 0.7 & 0.05 & 0.73 \\
\hline GS7P2 & G- \& G+ & 0.7 & 0.1 & 1.46 \\
\hline GS7P3 & G- \& G+ & 0.7 & 0.2 & 2.93 \\
\hline GS7P4 & G- \& G+ & 0.7 & 0.3 & 4.39 \\
\hline
\end{tabular}

Figure 2. (A) Complex coacervate (CC) phase diagram. In the twophase region a gradient from yellow to red is used to indicate the transition from a liquid dilute phase (left side) to a liquid CC phase (top right side) and finally to a solid CC phase (bottom right side). (B) Effect of polymer concentration: zoom in the liquid CC area, in which the black dots represent the analyzed samples, which phase separate into a dilute phase, shown as a light blue dot, and a complex coacervate phase, shown as a red dot. (C) Samples analyzed in strategy 2.

In the phase diagram (Figure 2A), two regions can be recognized: a one-phase region (white color) and a two-phase region (colored with a yellow-to-red gradient) in which phase separation into a dilute phase (on the left border of the diagram) and a complex coacervate phase (on the right border) occurs. In Figure $2 \mathrm{~B}$, we assume that the salt concentration is the same in the dilute and complex coacervate phase, in line with other reports in the literature. ${ }^{14}$ However, it should be mentioned that other researchers have found that salt concentrations are not the same in the two phases. ${ }^{41-43}$ The dilute phase is always a liquid aqueous solution (as indicated by the yellow color in Figure 2) at any salt concentration, while the complex coacervate phase transitions from a solid (red) to a liquid (orange) by increasing the added $\mathrm{NaCl}$ concentration. When surpassing the critical salt concentration (CSC), which in this system is between 0.8 and $0.85 \mathrm{M} \mathrm{NaCl}$, the electrostatic interactions are screened to such an extent that phase separation is prevented. At $0.7 \mathrm{M}$ $\mathrm{NaCl}$, a phase-separated material is obtained with a viscosity low enough to ensure injectability of the adhesive: therefore, this ionic strength is set as standard for all following experiments.

When increasing the polymer concentration while maintaining the salt concentration constant, the system (black dots in Figure 2B) always phase separates into the same two phases with the same polymer concentrations (blue and red dots in Figure $2 \mathrm{~B}$ ): the only factor that changes is the volumetric ratio between the two phases, with the complex coacervate phase percentage increasing as the initial polymer concentration increases. When the polymer concentration at mixing reaches the polymer concentration in the complex coacervate phase, the system enters a one-phase region: the polymer concentration at mixing is equal to the final one, meaning that an increase in the polymer concentration in the preparation stage leads to a final material with a higher polymer concentration. To reach a concentration that is high enough to access this region, several samples, shown in Figure $2 \mathrm{C}$ and indicated with black dots in Figure 2B, were prepared by varying the polymer concentration in the preparation stage. When preparing complex coacervates, it is more straightforward to express the starting polymer concentration in terms of total charged monomer concentration. However, that expression does not give a clear picture of the total amount of polymer present in solution: to facilitate the comparison with the amount of polymer present in the complex coacervate phase, those values have been transformed into total polymer percentages.

As shown in Figure 3A, over the whole range of polymer concentrations analyzed, complex coacervates with nearly the
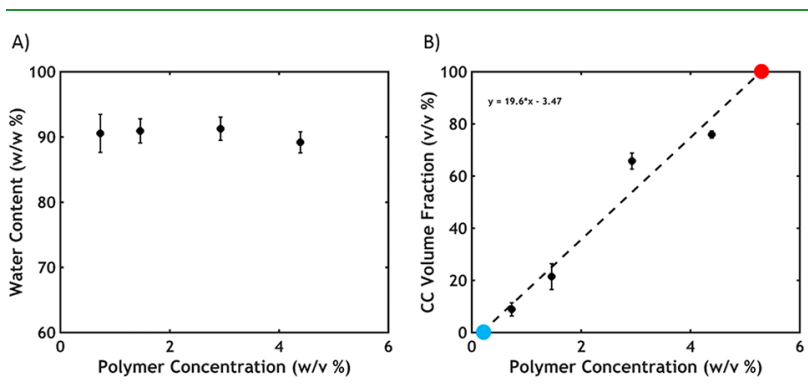

Figure 3. Effect of the polymer concentration on (A) water content and (B) complex coacervate volume fraction. The theoretical polymer concentrations of the dilute (blue dot) and complex coacervate phase (red dot) are obtained by extrapolating the regression line to $y=$ $100 \%$ and $y=0 \%$, respectively.

same water content (around 90\%), and consequently the same polymer concentration, are obtained. Figure $3 \mathrm{~B}$ indicates that the complex coacervate volume fraction increases linearly when increasing the polymer concentration, as expected.

However, contrary to our expectations, it was not possible to enter the one-phase region (and therefore increase the polymer concentration) because of solubility issues, especially concerning the anionic graft copolymer. When mixing the polyelec- 
A)

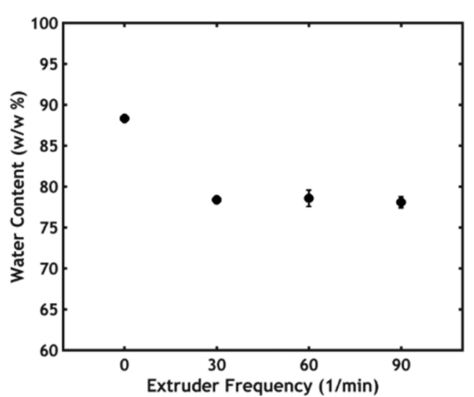

B)

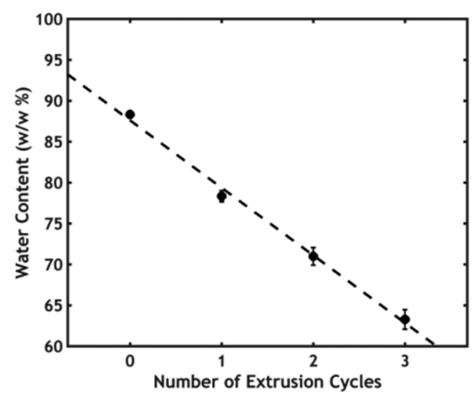

C)

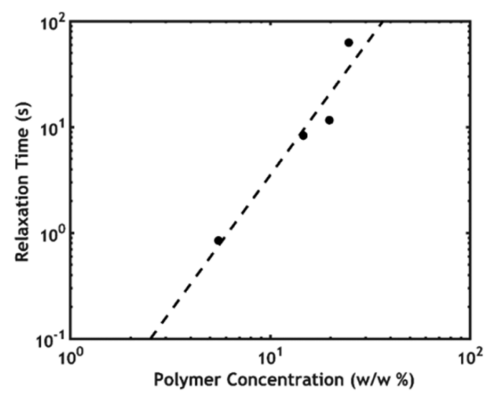

Figure 4. Water content as a function of (A) extruder frequency and (B) number of extrusion cycles. (C) Relaxation time plotted as a function of polymer concentration (the last point is not the real value but the relaxation time corresponding to the minimum frequency accessed in the experiment).

trolytes at a 1:1 charge ratio, the maximum reachable total polymer content is $4.39 \% \mathrm{w} / \mathrm{v}$, which, as shown in Figure $3 \mathrm{~B}$, is lower than the polymer concentration in the complex coacervate phase (around $5.3 \% \mathrm{w} / \mathrm{v}$ ), obtained by extrapolating the regression line in Figure $3 \mathrm{~B}$ to $100 \%$ (border between the two-phase and one-phase region). The value obtained with this method is comparable to the one obtained by performing a thermogravimetric analysis (TGA) on the complex coacervate phase $(5.5 \% \mathrm{w} / \mathrm{v})$, highlighting the validity of the phase diagram. In conclusion, increasing the polymer concentration in the preparation stage is not an effective strategy to raise the polymer content in the complex coacervate phase.

Strategy 3: Extrusion. As a third strategy, we used a miniextruder to mechanically force water out of the adhesive. The added salt concentration and the total charged monomer concentration are $0.7 \mathrm{M} \mathrm{NaCl}$ and $0.05 \mathrm{M}$, respectively. After complex coacervate formation, the dilute phase is removed. The complex coacervate is heated above its LCST and inserted through an inlet on top of the screws in the extrusion chamber. Then, the material is submitted to an extrusion cycle at $50{ }^{\circ} \mathrm{C}$. At first, the material is moved forward in the chamber by the shearing forces of the extruder screws; its progression is then stopped when the whole sample has passed through the screws, with the complex coacervate being solidified in an inner reservoir (Figure S5). After $3 \mathrm{~min}$ in the chamber, the material is removed and stored in the refrigerator overnight, and if required, the following day another cycle is performed (up to a maximum of 3). After one cycle, expelled water, separated from the material, is visible in the extruded chamber (Figure S5), indicating that this strategy is effective in reducing the water content and, therefore, in increasing the polymer concentration of the material. To study the process in more detail, the effect of the extruder frequency and of the number of extrusion cycles are investigated (Figure 4, Figures S6 and S7).

A drop in water content from $88 \%$ to $78 \%$ (Figure $4 \mathrm{~A}$ ), giving rise to an increase in viscoelastic moduli $G^{\prime}$ and $G^{\prime \prime}$ (Figure S6), is observed after one extrusion cycle, independently of extruder frequency. Therefore, for the remaining measurements, the extruder frequency was set to $30 / \mathrm{min}$. In contrast, increasing the number of extrusion cycles, after homogenizing the material in the fridge overnight between two successive cycles, significantly influences the complex coacervate phase properties. The water content decreases at a higher number of cycles, reaching $63 \%$ after three extrusion processes (Figure 4B).
Differently from the extruder frequency, a higher number of cycles profoundly affect the rheological behavior: the as-made material shows typical features of a viscous liquid at a temperature below the PNIPAM LCST, with both moduli frequency dependent (Figure S7A). $G^{\prime}$ overcomes $G^{\prime \prime}$ at a crossover frequency $\omega_{\mathrm{c}}$ of $7.36 \mathrm{rad} / \mathrm{s}$, corresponding to a relaxation time $\tau=1 / \omega_{\mathrm{c}}=0.85 \mathrm{~s}$. After one extrusion cycle, the water content drops to $78 \%$, with the complex coacervates still showing liquid behavior. However, the moduli become less frequency dependent and increase. The relaxation time increases by an order of magnitude to $\tau=8.33 \mathrm{~s}$. This behavior becomes more obvious at a higher number of cycles: after three extrusion cycles, the crossover frequency is not detectable in the window of frequencies analyzed, meaning that $\tau \geq 62.5 \mathrm{~s}$. This is evidence of a higher polymer concentration in the material which slows down chain relaxation, in accordance with the sticky Rouse model, which has been adopted to describe chain dynamics in complex coacervates $^{40,44}$ and which predicts a power-law increase of the relaxation time with the polymer volume fraction, as observed in Figure 4C.

When performing a temperature switch by heating the sample above the LCST of PNIPAM (between 10 and $35{ }^{\circ} \mathrm{C}$, Figures S9 and S10), the collapse of the thermoresponsive chains is promoted: as a result, all samples acquire characteristics of soft solids, with $G^{\prime}$ higher than $G^{\prime \prime}$, and both frequency independent (Figure S7B). $G^{\prime}$ shows a power-law increase as a function of the polymer concentration (Figure 5A), highlighting that the extrusion process is effective in stiffening the material by increasing the polymer concentration: this is in
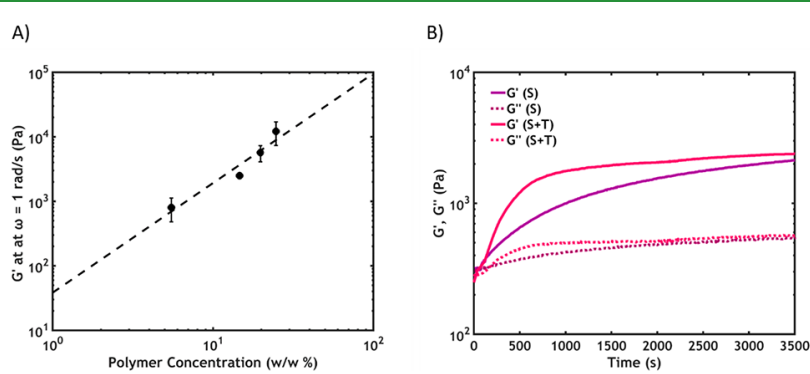

Figure 5. Solidification upon temperature or salt switch. (A) Storage modulus (recorded at $\omega=1 \mathrm{rad} / \mathrm{s}$ and $T=50{ }^{\circ} \mathrm{C}$ ) as a function of polymer concentration. (B) Time sweeps after one extrusion cycle when performing a salt $(S)$ or a combined salt and temperature $(\mathrm{S}+\mathrm{T})$ switch. 
A)

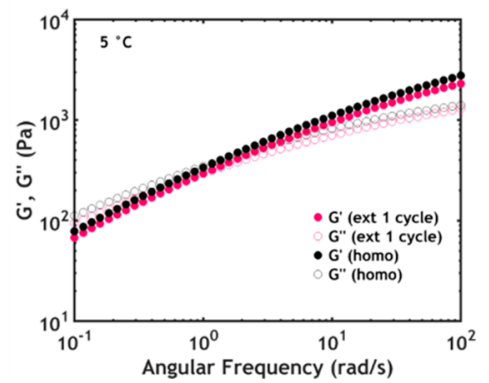

B)

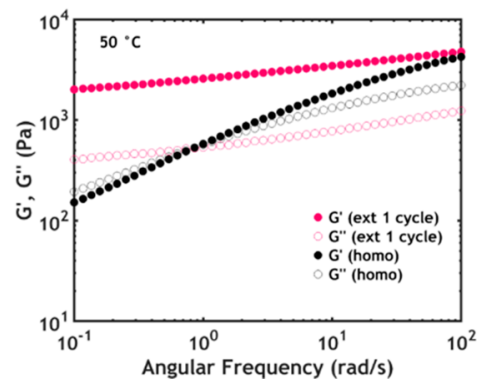

C)

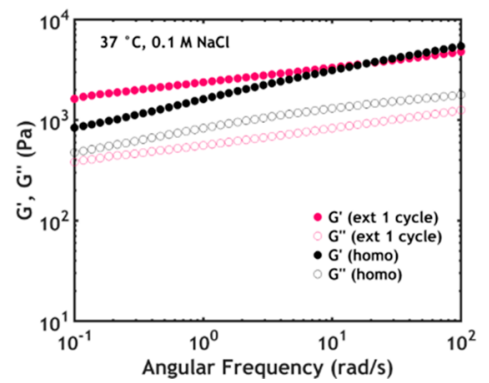

Figure 6. Rheological properties of extruded and homopolymer complex coacervates: (A) frequency sweeps performed at $5{ }^{\circ} \mathrm{C}$, (B) frequency sweep performed at $50{ }^{\circ} \mathrm{C}$, and (C) frequency sweeps performed after a combined temperature and salt switch.

accordance with the theory of rubber elasticity, which states that the storage modulus is proportional to the amount of elastically active chains per unit volume. ${ }^{45}$

The adhesive can also be reinforced by performing a salt switch, namely, by exposing the material to an ionic strength gradient. When the sample is exposed to a lower ionic strength environment $(0.1 \mathrm{M} \mathrm{NaCl})$, the excess salt ions diffuse out of the adhesive, strengthening the electrostatic interactions between the polyelectrolyte chains. This promotes a reinforcement of the material over time, with the moduli progressively increasing and heading toward a plateau after $1 \mathrm{~h}$ (Figure 5B). When applying a combined salt and temperature switch, different kinetics are observed (Figure 5B). The rheological properties increase more abruptly during the first stages of the transition due to the immediate collapse (response) of the thermoresponsive PNIPAM chains when exposed to a medium with a higher temperature than the LCST. This process is much faster than ion diffusion from a confined region. In contrast, for the salt switch, a contact time of $1 \mathrm{~h}$ is required for the full setting of the material. ${ }^{22}$ At the end of the transition, the complex coacervate phase has the characteristics of a soft elastic solid, with increasing moduli for samples with a higher number of extrusion cycles (Figure S11A). The obtained values (Figure S11B) are similar to the ones obtained after a single temperature switch: this means that the total number of nodes is almost the same. However, in this case, both PNIPAM-PNIPAM and electrostatic interactions contribute to the final moduli, while when a temperature switch is applied, the formed network results mainly from the collapse of the thermoresponsive chains.

Figure 6 shows the rheological properties of the sample obtained after one extrusion cycle and of the homopolymer complex coacervate: despite having a different preparation history, the two materials have the same water content $(78 \%$ for both samples).

At temperatures below PNIPAM LCST, the polymer concentration seems to dictate the rheological properties, with both samples showing a similar behavior (Figure 6A). However, the presence of PNIPAM is crucial above the LCST: when heated (Figure 6B), the extruded sample turns into a gel because of the collapse of the PNIPAM chains which abruptly slows down the relaxation processes, while in the homopolymer complex coacervates, no considerable variation is visible due to the absence of any temperature-sensitive unit. However, both samples show a transition in response to a combined temperature-salt switch, mimicking the conditions that the sample would experience in a physiological environment (Figure 6C). Both materials turn into soft gels, with $G^{\prime}$ overcoming $G^{\prime \prime}$ at any analyzed frequency. However, the moduli of the homopolymer complex coacervates are more frequency dependent, meaning that the crossover frequency (not detectable because it is out of the frequency range analyzed) is anticipated to be higher and the relaxation time will therefore be lower. This indicates that despite a similar polymer concentration, the presence of the PNIPAM units allows the formation of a soft elastic network which is more effective in slowing down chain relaxation.

The underwater adhesive properties of the extruded graft samples are determined by using the setup developed by Sudre et al. $^{46}$ In Figure $7 \mathrm{~A}$, the effect of a combined temperature-salt switch is reported for samples that were exposed to different extrusion cycles.
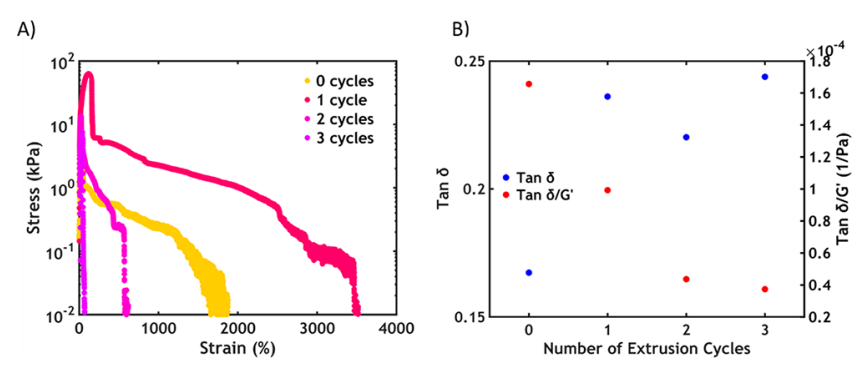

Figure 7. (A) Underwater adhesion performance after application of a temperature-salt switch at a nominal strain rate of $0.2 \mathrm{~s}^{-1}$. (B) Plot of $\tan \delta$ and $\tan \delta / G^{\prime}$ recorded with a rheometer at $\omega=1 \mathrm{rad} / \mathrm{s}$ (comparable to the strain rate in the tack measurement) as a function of the number of extrusion cycles.

Compared to the unprocessed sample ( 0 cycles), the adhesive that has been submitted to one extrusion cycle shows a much higher adhesion energy, with both an increase in the peak stress $(60 \mathrm{kPa})$ and a maximum strain $(3500 \%)$. The mode of failure is the same in both samples, with the material failing cohesively, leaving residues on both retracting surfaces. The higher polymer concentration reinforces the material, enabling a higher resistance to an applied stress: this might be ascribed to a combination of increased stiffness, as observed in Figure $5 \mathrm{~A}$, and dissipation, characterized by the damping factor $\tan \delta\left(G^{\prime \prime} / G^{\prime}\right)$, which shows a $50 \%$ increase after one extrusion cycle (Figure 7B). However, a further increase in polymer concentration, obtained when performing additional extrusion cycles, leads to a decrease in the adhesive performance: despite showing a higher peak stress than the unprocessed sample, the strain at break decreases significantly. The mode of failure also changes, with the material now failing adhesively, without 
residues on the probe. This suggests a much less viscoelastic and more elastic gel-like behavior. In adhesion science, this is generally quantified by the ratio of the damping factor to the storage modulus, employed as a gauge of expected adhesive performance. ${ }^{47}$ An excessively high value of $G^{\prime}$, relative to the dissipative character of the adhesive, will prevent cavity formation in the vertical direction: ${ }^{47}$ this means that when the material is too stiff and too elastic, i.e., a too low value of $\tan \delta / G^{\prime}$ (Figure $7 \mathrm{~B}$ ), resistance to interfacial crack propagation is too low, and the material fails adhesively at low strain. ${ }^{47}$ Therefore, an optimal polymer concentration is needed for a balance between cohesive and adhesive properties. $^{48}$

Another requirement that an underwater adhesive should meet is the dimensional stability in submerged conditions. ${ }^{25,49}$ When performing an extrusion process, the complex coacervate phase is brought in an out of equilibrium condition and, if submerged, could likely reabsorb the water which has been previously removed. Swelling experiments were therefore performed on the samples in conditions mimicking the physiological environment $\left(T=37{ }^{\circ} \mathrm{C},[\mathrm{NaCl}]=0.1 \mathrm{M}\right.$, $\mathrm{pH}=7.0$ ) over a period of $1 \mathrm{~h}$ to check the water uptake/ release in the time scale of the experiment (Figure $8 \mathrm{~A}$ ). The
A)

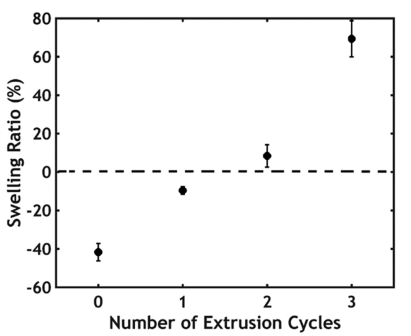

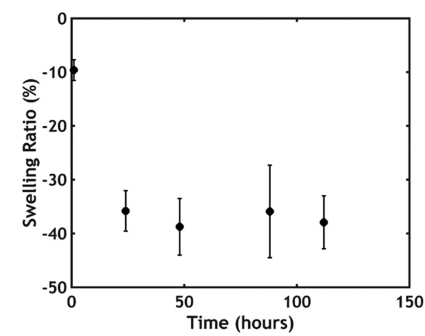

Figure 8. (A) Swelling ratio versus the number of extrusion cycles. The dashed line represents the border between swelling (above the line) and shrinking (below the line). (B) Swelling ratio as a function of time for the sample extruded one time in physiological conditions $\left(T=37{ }^{\circ} \mathrm{C},[\mathrm{NaCl}]=0.1 \mathrm{M}, \mathrm{pH}=7.0\right)$.

unprocessed sample, with the highest water content, shows a negative swelling ratio: this is an indication of shrinking, mainly due to the collapse of the PNIPAM chains, as already observed in other work on thermoresponsive adhesives, ${ }^{25}$ and to the contraction of the polyelectrolyte matrix at lower ionic strength. Additionally, the sample turns white (Figure S12) evidence that water also remains trapped within the material, forming a porous structure, as already proposed in our previous work. ${ }^{21-23}$

The swelling ratio increases as a function of extrusion cycles: the samples submitted to one extrusion cycle shows a higher dimensional stability, exhibiting a swelling ratio close to zero (Figure 8A), similar to what is observed in hydrophobic tissue adhesives. ${ }^{29,30}$ This means that the water removed through the extrusion process is not reabsorbed by the sample (no swelling is observed over a 5 day period, Figure $8 \mathrm{~B}$ ). However, when the amount of extrusion cycles is further increased, the material swells due to water sorption (Figure S12A), which might also contribute to the decrease in the adhesive performance as seen in Figure 7, as reported for PEG-based tissue adhesives. ${ }^{28}$ When decreasing the amount of water within the material, the adhesive, after setting, becomes less opaque (Figure S12)- evidence of a lower amount and/or smaller size pores containing trapped water within the material. ${ }^{36}$

Therefore, the optimal balance between adhesive and cohesive properties, together with a proper dimensional stability, leads to a large enhancement of the adhesion performance in the sample submitted to only one extrusion cycle.

A poorer adhesive performance is observed when submitting the samples only to a temperature trigger (Figure 9A). Despite
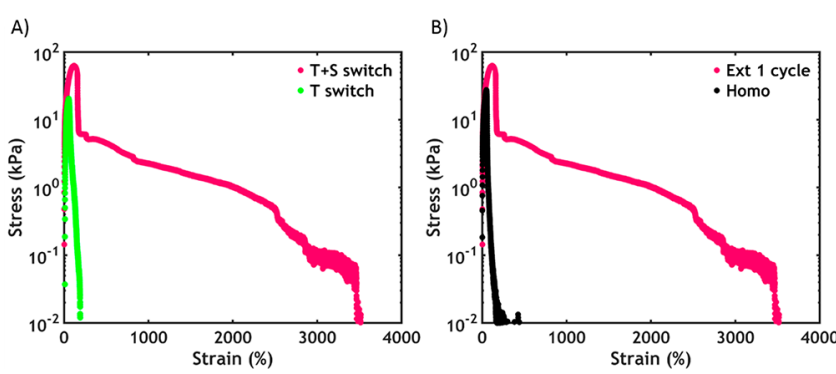

Figure 9. Underwater adhesive performance of the sample after one extrusion cycle after application of a temperature-salt switch compared to (A) the application of a single temperature switch and (B) the performance of the homopolymer complex coacervate upon a combined trigger.

obtaining similar moduli when reinforcing the material with a single or a combined trigger (Figure 5A and Figure S11B), the sample fails in an adhesive fashion at a much lower strain when applying only a temperature trigger: this could be correlated to the size of the polymers involved in the reinforcing mechanism. When just a temperature trigger is performed, only the short PNIPAM chains are collapsed, forming domains of a small size. Thereby, when applying a detaching stress, the chains can be stretched only to a small extent. By contrast, by decreasing the ionic strength, also the electrostatic interactions between the long backbones are activated. To break the sample, also these additional bonds need to be disrupted and the adhesive can be stretched to a higher extent before failure, resulting in a higher toughness. Additionally, this behavior might also be ascribed to the more viscoelastic character of the material after a combined switch, as confirmed by the slightly higher values of the damping factor and of $\tan \delta / G^{\prime}$ as compared to values obtained for a single temperature switch (Figure S13A).

Similarly, when probing the homopolymer counterpart with the same water content in physiological conditions, we observed an adhesive failure at low strain (Figure 9B). This behavior is surprising since, as observed in Figure S13B, the homopolymer complex coacervates have a more dissipative character than the extruded sample. In this case, the linear rheology properties fail to predict the nonlinear behavior observed in the probe-tack test. The improved adhesive performance shown by the extruded sample might indicate that the presence of two types of interactions in the same material (PNIPAM nodes and electrostatic interactions) favors an increase in toughness (not detectable via linear rheology), which might be related to the variety of bond strength present in the adhesive, as observed for polyampholyte hydrogels and double networks. ${ }^{50-52}$ Additionally, the higher (in absolute value) swelling ratio $(-23.4 \%)$ reported in our previous work $^{22}$ for homopolymer complex coacervates measured in similar conditions might affect the adhesive properties: a higher amount of released water might weaken the interfacial 
interactions with the probe, resulting in a lower dimensional stability and a worse adhesive performance compared to the extruded samples, which show a lower swelling ratio (-9.6\%).

Lastly, in Figure 10 the work of adhesion $\left(W_{\text {adh }}\right)$, obtained from the area below the stress-strain curves, is plotted versus the amount of extrusion cycles.

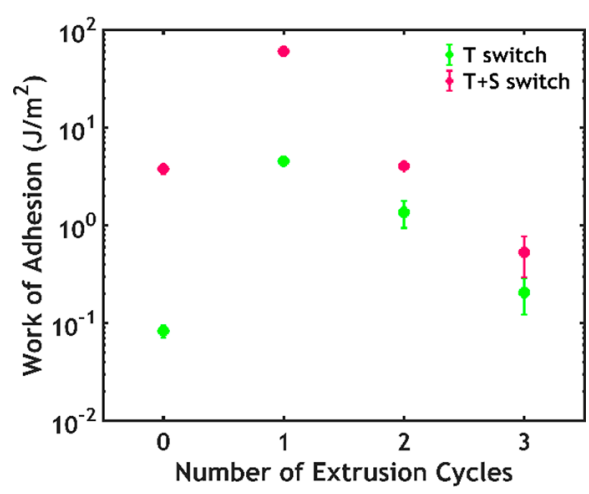

Figure 10. Work of adhesion versus the amount of extrusion cycles and versus the applied trigger.

After one extrusion cycle, when performing a combined switch, the work of adhesion increases from 3.8 to $60.6 \mathrm{~J} / \mathrm{m}^{2}$, which is much higher than the one shown by the homopolymer counterpart $\left(4.7 \mathrm{~J} / \mathrm{m}^{2}\right.$, not shown in the figure) and the one obtained after performing a temperature switch only $\left(4.6 \mathrm{~J} / \mathrm{m}^{2}\right)$. When further raising the number of extrusion cycles, the work of adhesion drastically decreases, with a similar trend observed for both triggers. An optimum underwater performance is obtained when a porous structure is preserved, the adhesive and cohesive properties are well balanced, and the in situ swelling is limited.

\section{CONCLUSIONS}

Polymer concentration strongly affects the mechanical properties of an adhesive. PNIPAM-functionalized complex coacervates exhibit a high water content, which inevitably affects the moduli and the adhesive properties of the material. Several routes have been adopted in this work to optimize the water content: among the attempted strategies, extrusion is the most effective in increasing the polymer concentration within the complex coacervate phase.

While a higher number of extrusion cycles does lead to a progressive enhancement of the dynamic moduli, the underwater $W_{\text {adh }}$ initially benefits from the higher polymer concentration within the material but then drastically drops when the water content is further decreased. By carefully tuning the polymer concentration, promising underwater adhesion data is achieved, reaching an adhesive strength of $60 \mathrm{kPa}$ and work of adhesion of $60 \mathrm{~J} / \mathrm{m}^{2}$ : these values are higher than those reported for commercial adhesives measured underwater, such as fibrin glues, and comparable to the highest values reported for bioinspired adhesives tested in similar conditions. $^{53,54}$ However, differently from those materials, which are already in the solid state before application or need to be solidified in situ with external agents, this glue sets immediately when released in physiological conditions, experiencing an environmentally triggered phase transition. Further studies are required to systematically address the role of the microstructure on the adhesion properties.

\section{ASSOCIATED CONTENT}

\section{(s) Supporting Information}

The Supporting Information is available free of charge at https://pubs.acs.org/doi/10.1021/acsapm.0c00185.

Experimental section, synthesis details of the polymers, additional rheology and swelling data (PDF)

\section{AUTHOR INFORMATION}

\section{Corresponding Author}

Marleen Kamperman - Laboratory of Physical Chemistry and Soft Matter, Wageningen University \& Research, 6708 WE Wageningen, The Netherlands; Polymer Science, Zernike Institute for Advanced Materials, University of Groningen, 9747 AG Groningen, The Netherlands; 10 orcid.org/0000-00020520-4534; Email: marleen.kamperman@rug.nl

\section{Authors}

Marco Dompé - Laboratory of Physical Chemistry and Soft Matter, Wageningen University \& Research, 6708 WE Wageningen, The Netherlands

Mehdi Vahdati - Soft Matter Sciences and Engineering, ESPCI Paris, PSL University, Sorbonne University, CNRS, F-75005 Paris, France

Froukje van Ligten - Laboratory of Physical Chemistry and Soft Matter, Wageningen University \& Research, 6708 WE Wageningen, The Netherlands

Francisco J. Cedano-Serrano - Soft Matter Sciences and Engineering, ESPCI Paris, PSL University, Sorbonne University, CNRS, F-75005 Paris, France; 이이이.org/0000-0001-97922551

Dominique Hourdet - Soft Matter Sciences and Engineering, ESPCI Paris, PSL University, Sorbonne University, CNRS, F75005 Paris, France; 이이이.org/0000-0002-0328-7014

Costantino Creton - Soft Matter Sciences and Engineering, ESPCI Paris, PSL University, Sorbonne University, CNRS, F75005 Paris, France; 이이이.org/0000-0002-0177-9680

Marco Zanetti - Department of Chemistry and NIS Centre, University of Turin, Torino 10125, Italy

Pierangiola Bracco - Department of Chemistry and NIS Centre, University of Turin, Torino 10125, Italy

Jasper van der Gucht - Laboratory of Physical Chemistry and Soft Matter, Wageningen University \& Research, 6708 WE Wageningen, The Netherlands; 1 orcid.org/0000-0001-55258322

Thomas Kodger - Laboratory of Physical Chemistry and Soft Matter, Wageningen University \& Research, 6708 WE Wageningen, The Netherlands

Complete contact information is available at:

https://pubs.acs.org/10.1021/acsapm.0c00185

\section{Funding}

The project received funding from the European Union's Horizon 2020 research and innovation program under the Marie Sklodowska-Curie grant agreement no. 642861. J.v.d.G. acknowledges the European Research Council for financial support (ERC Consolidator grant Softbreak).

\section{Notes}

The authors declare no competing financial interest.

\section{REFERENCES}

(1) Mazza, P. P. A.; Martini, F.; Sala, B.; Magi, M.; Colombini, M. P.; Giachi, G.; Landucci, F.; Lemorini, C.; Modugno, F.; Ribechini, E. 
A new Palaeolithic discovery: tar-hafted stone tools in a European Mid-Pleistocene bone-bearing bed. Journal of Archaeological Science 2006, 33 (9), 1310-1318.

(2) Lauto, A.; Mawad, D.; Foster, L. J. R. Adhesive biomaterials for tissue reconstruction. J. Chem. Technol. Biotechnol. 2008, 83 (4), 464472.

(3) Bhagat, V.; Becker, M. L. Degradable Adhesives for Surgery and Tissue Engineering. Biomacromolecules 2017, 18 (10), 3009-3039.

(4) Bouten, P. J. M.; Zonjee, M.; Bender, J.; Yauw, S. T. K.; van Goor, H.; van Hest, J. C. M.; Hoogenboom, R. The chemistry of tissue adhesive materials. Prog. Polym. Sci. 2014, 39 (7), 1375-1405.

(5) Mehdizadeh, M.; Yang, J. Design Strategies and Applications of Tissue Bioadhesives. Macromol. Biosci. 2013, 13 (3), 271-288.

(6) Bré, L. P.; Zheng, Y.; Pêgo, A. P.; Wang, W. Taking tissue adhesives to the future: from traditional synthetic to new biomimetic approaches. Biomater. Sci. 2013, 1 (3), 239-253.

(7) Rahimnejad, M.; Zhong, W. Mussel-inspired hydrogel tissue adhesives for wound closure. RSC Adv. 2017, 7 (75), 47380-47396.

(8) Stewart, R. J.; Weaver, J. C.; Morse, D. E.; Waite, J. H. The tube cement of Phragmatopoma californica: a solid foam. J. Exp. Biol. 2004, 207 (26), 4727-4734.

(9) Waite, J. H.; Andersen, N. H.; Jewhurst, S.; Sun, C. Mussel Adhesion: Finding the Tricks Worth Mimicking. J. Adhes. 2005, 81 (3-4), 297-317.

(10) Walker, G. The histology, histochemistry and ultrastructure of the cement apparatus of three adult sessile barnacles, Elminius modestus, Balanus balanoides and Balanus hameri. Mar. Biol. 1970, 7 (3), 239-248.

(11) Stewart, R. J.; Wang, C. S.; Shao, H. Complex coacervates as a foundation for synthetic underwater adhesives. Adv. Colloid Interface Sci. 2011, 167 (1-2), 85-93.

(12) Stewart, R. J.; Wang, C. S.; Song, I. T.; Jones, J. P. The role of coacervation and phase transitions in the sandcastle worm adhesive system. Adv. Colloid Interface Sci. 2017, 239, 88-96.

(13) Gucht, J. v. d.; Spruijt, E.; Lemmers, M.; Cohen Stuart, M. A. Polyelectrolyte complexes: Bulk phases and colloidal systems. J. Colloid Interface Sci. 2011, 361 (2), 407-422.

(14) Spruijt, E.; Westphal, A. H.; Borst, J. W.; Cohen Stuart, M. A.; van der Gucht, J. Binodal Compositions of Polyelectrolyte Complexes. Macromolecules 2010, 43 (15), 6476-6484.

(15) Kaur, S.; Weerasekare, G. M.; Stewart, R. J. Multiphase Adhesive Coacervates Inspired by the Sandcastle Worm. ACS Appl. Mater. Interfaces 2011, 3 (4), 941-944.

(16) Shao, H.; Stewart, R. J. Biomimetic Underwater Adhesives with Environmentally Triggered Setting Mechanisms. Adv. Mater. 2010, 22 (6), 729-733.

(17) Ahn, B. K.; Das, S.; Linstadt, R.; Kaufman, Y.; MartinezRodriguez, N. R.; Mirshafian, R.; Kesselman, E.; Talmon, Y.; Lipshutz, B. H.; Israelachvili, J. N.; Waite, J. H. High-performance musselinspired adhesives of reduced complexity. Nat. Commun. 2015, 6, 8663.

(18) Seo, S.; Das, S.; Zalicki, P. J.; Mirshafian, R.; Eisenbach, C. D.; Israelachvili, J. N.; Waite, J. H.; Ahn, B. K. Microphase Behavior and Enhanced Wet-Cohesion of Synthetic Copolyampholytes Inspired by a Mussel Foot Protein. J. Am. Chem. Soc. 2015, 137 (29), 9214-9217. (19) Zhao, Q.; Lee, D. W.; Ahn, B. K.; Seo, S.; Kaufman, Y.; Israelachvili, J. N.; Waite, J. H. Underwater contact adhesion and microarchitecture in polyelectrolyte complexes actuated by solvent exchange. Nat. Mater. 2016, 15, 407.

(20) Zhang, C.; Huang, J.; Zhang, J.; Liu, S.; Cui, M.; An, B.; Wang, X.; Pu, J.; Zhao, T.; Fan, C.; Lu, T. K.; Zhong, C. Engineered Bacillus subtilis biofilms as living glues. Mater. Today 2019, 28, 40-48.

(21) Dompé, M.; Cedano-Serrano, F. J.; Heckert, O.; van den Heuvel, N.; van der Gucht, J.; Tran, Y.; Hourdet, D.; Creton, C.; Kamperman, M. Thermoresponsive Complex Coacervate-Based Underwater Adhesive. Adv. Mater. 2019, 31 (21), 1808179.

(22) Dompe, M.; Cedano-Serrano, F. J.; Vahdati, M.; Westerveld, L.; Hourdet, D.; Creton, C.; der Gucht, J.; Kodger, T.; Kamperman, M.
Underwater Adhesion of Multiresponsive Complex Coacervates. $A d v$. Mater. Interfaces 2020, 7, 1901785.

(23) Dompé, M.; Cedano-Serrano, F. J.; Vahdati, M.; Sidoli, U.; Heckert, O.; Synytska, A.; Hourdet, D.; Creton, C.; Van der Gucht, J.; Kodger, T.; Kamperman, M. Tuning the Interactions in Multiresponsive Complex Coacervate-Based Underwater Adhesives. Int. J. Mol. Sci. 2020, 21, 100.

(24) Kelmansky, R.; McAlvin, B. J.; Nyska, A.; Dohlman, J. C.; Chiang, H. H.; Hashimoto, M.; Kohane, D. S.; Mizrahi, B. Strong tissue glue with tunable elasticity. Acta Biomater. 2017, 53, 93-99.

(25) Barrett, D. G.; Bushnell, G. G.; Messersmith, P. B. Mechanically Robust, Negative-Swelling, Mussel-Inspired Tissue Adhesives. Adv. Healthcare Mater. 2013, 2 (5), 745-755.

(26) Lee, G.; Lee, C. K.; Bynevelt, M. DuraSeal-Hematoma: Concealed Hematoma Causing Spinal Cord Compression. Spine 2010, 35 (25), E1522-E1524.

(27) Mulder, M.; Crosier, J.; Dunn, R. Cauda Equina Compression by Hydrogel Dural Sealant After a Laminotomy and Discectomy: Case Report. Spine 2009, 34 (4), E144-E148.

(28) Campbell, P. K.; Bennett, S. L.; Driscoll, A.; Sawhney, A. S. Evaluation of absorbable surgical sealants: in vitro testing. https:// pdfs.se manticscholar.org/0 e $72 /$ 159a6027168d8ecb11dcd2375ad692c30ab3.pdf, 2005.

(29) Lang, N.; Pereira, M. J.; Lee, Y.; Friehs, I.; Vasilyev, N. V.; Feins, E. N.; Ablasser, K.; O’Cearbhaill, E. D.; Xu, C.; Fabozzo, A.; Padera, R.; Wasserman, S.; Freudenthal, F.; Ferreira, L. S.; Langer, R.; Karp, J. M.; del Nido, P. J. A Blood-Resistant Surgical Glue for Minimally Invasive Repair of Vessels and Heart Defects. Sci. Transl. Med. 2014, 6 (218), No. 218ra6.

(30) Nijst, C. L. E.; Bruggeman, J. P.; Karp, J. M.; Ferreira, L.; Zumbuehl, A.; Bettinger, C. J.; Langer, R. Synthesis and Characterization of Photocurable Elastomers from Poly(glycerol-co-sebacate). Biomacromolecules 2007, 8 (10), 3067-3073.

(31) Yuk, H.; Varela, C. E.; Nabzdyk, C. S.; Mao, X.; Padera, R. F.; Roche, E. T.; Zhao, X. Dry double-sided tape for adhesion of wet tissues and devices. Nature 2019, 575 (7781), 169-174.

(32) Feldstein, M. M. Molecular Nature of Pressure-Sensitive Adhesion. In Fundamentals of Pressure Sensitivity; Benedek, I., Feldstein, M. M., Eds.; Taylor \& Francis Group, LLC: Boca Raton, FL, 2008.

(33) Chalykh, A. A.; Chalykh, A. E.; Novikov, M. B.; Feldstein, M. M. Pressure-sensitive adhesion in the blends of poly(N-vinyl pyrrolidone) and poly(ethylene glycol) of disparate chain lengths. J. Adhes. 2002, 78 (8), 667-694.

(34) Roos, A.; Creton, C.; Novikov, M. B.; Feldstein, M. M. Viscoelasticity and tack of poly(vinyl pyrrolidone)-poly(ethylene glycol) blends. J. Polym. Sci., Part B: Polym. Phys. 2002, 40 (20), 2395-2409.

(35) Fu, J.; Wang, Q.; Schlenoff, J. B. Extruded Superparamagnetic Saloplastic Polyelectrolyte Nanocomposites. ACS Appl. Mater. Interfaces 2015, 7 (1), 895-901.

(36) Shamoun, R. F.; Reisch, A.; Schlenoff, J. B. Extruded Saloplastic Polyelectrolyte Complexes. Adv. Funct. Mater. 2012, 22 (9), 19231931.

(37) Wang, Q.; Schlenoff, J. B. Tough strained fibers of a polyelectrolyte complex: pretensioned polymers. RSC Adv. 2014, 4 (87), 46675-46679.

(38) Zhang, Y.; Furyk, S.; Bergbreiter, D. E.; Cremer, P. S. Specific Ion Effects on the Water Solubility of Macromolecules: PNIPAM and the Hofmeister Series. J. Am. Chem. Soc. 2005, 127 (41), 1450514510 .

(39) Hariri, H. H.; Lehaf, A. M.; Schlenoff, J. B. Mechanical Properties of Osmotically Stressed Polyelectrolyte Complexes and Multilayers: Water as a Plasticizer. Macromolecules 2012, 45 (23), 9364-9372.

(40) Spruijt, E.; Cohen Stuart, M. A.; van der Gucht, J. Linear Viscoelasticity of Polyelectrolyte Complex Coacervates. Macromolecules 2013, 46 (4), 1633-1641. 
(41) Li, L.; Srivastava, S.; Andreev, M.; Marciel, A. B.; de Pablo, J. J.; Tirrell, M. V. Phase Behavior and Salt Partitioning in Polyelectrolyte Complex Coacervates. Macromolecules 2018, 51 (8), 2988-2995.

(42) Perry, S. L.; Sing, C. E. PRISM-Based Theory of Complex Coacervation: Excluded Volume versus Chain Correlation. Macromolecules 2015, 48 (14), 5040-5053.

(43) Qin, J.; Priftis, D.; Farina, R.; Perry, S. L.; Leon, L.; Whitmer, J.; Hoffmann, K.; Tirrell, M.; de Pablo, J. J. Interfacial Tension of Polyelectrolyte Complex Coacervate Phases. ACS Macro Lett. 2014, 3 (6), 565-568.

(44) Spruijt, E.; Sprakel, J.; Lemmers, M.; Stuart, M. A. C.; van der Gucht, J. Relaxation Dynamics at Different Time Scales in Electrostatic Complexes: Time-Salt Superposition. Phys. Rev. Lett. 2010, 105 (20), 208301.

(45) Flory, P. J. Principles of Polymer Chemistry; Cornell University Press: Ithaca, NY, 1953.

(46) Sudre, G.; Olanier, L.; Tran, Y.; Hourdet, D.; Creton, C. Reversible adhesion between a hydrogel and a polymer brush. Soft Matter 2012, 8 (31), 8184-8193.

(47) Wang, T.; Lei, C.-H.; Dalton, A. B.; Creton, C.; Lin, Y.; Fernando, K. A. S.; Sun, Y.-P.; Manea, M.; Asua, J. M.; Keddie, J. L. Waterborne, Nanocomposite Pressure-Sensitive Adhesives with High Tack Energy, Optical Transparency, and Electrical Conductivity. Adv. Mater. 2006, 18 (20), 2730-2734.

(48) Deplace, F.; Carelli, C.; Mariot, S.; Retsos, H.; Chateauminois, A.; Ouzineb, K.; Creton, C. Fine Tuning the Adhesive Properties of a Soft Nanostructured Adhesive with Rheological Measurements. J. Adhes. 2009, 85 (1), 18-54.

(49) Jones, J. P.; Sima, M.; O’Hara, R. G.; Stewart, R. J. Water-Borne Endovascular Embolics Inspired by the Undersea Adhesive of Marine Sandcastle Worms. Adv. Healthcare Mater. 2016, 5 (7), 795-801.

(50) Sun, T. L.; Kurokawa, T.; Kuroda, S.; Ihsan, A. B.; Akasaki, T.; Sato, K.; Haque, M. A.; Nakajima, T.; Gong, J. P. Physical hydrogels composed of polyampholytes demonstrate high toughness and viscoelasticity. Nat. Mater. 2013, 12, 932.

(51) Gong, J. P. Why are double network hydrogels so tough? Soft Matter 2010, 6 (12), 2583-2590.

(52) Sun, J.-Y.; Zhao, X.; Illeperuma, W. R. K.; Chaudhuri, O.; Oh, K. H.; Mooney, D. J.; Vlassak, J. J.; Suo, Z. Highly stretchable and tough hydrogels. Nature 2012, 489 (7414), 133-136.

(53) Clancy, S. K.; Sodano, A.; Cunningham, D. J.; Huang, S. S.; Zalicki, P. J.; Shin, S.; Ahn, B. K. Marine Bioinspired Underwater Contact Adhesion. Biomacromolecules 2016, 17 (5), 1869-1874.

(54) Rao, P.; Sun, T. L.; Chen, L.; Takahashi, R.; Shinohara, G.; Guo, H.; King, D. R.; Kurokawa, T.; Gong, J. P. Tough Hydrogels with Fast, Strong, and Reversible Underwater Adhesion Based on a Multiscale Design. Adv. Mater. 2018, 30 (32), 1801884. 